\title{
Individual difference in prepulse inhibition does not predict spatial learning and memory performance in $\mathrm{C} 57 \mathrm{BL} / 6$ mice
}

\author{
Daria Peleg-Raibstein $^{1,2} \cdot$ Singer Philipp ${ }^{1,3} \cdot$ Joram Feldon $^{1} \cdot$ Benjamin K. Yee $^{1,3}$
}

Published online: 17 April 2015

(C) Psychonomic Society, Inc. 2015

\begin{abstract}
The startle reflex to an intense acoustic pulse stimulus is attenuated if the pulse stimulus is shortly preceded by a weak non-startling prepulse stimulus. This attenuation of the startle reflex represents a form of pre-attentional sensory gating known as prepulse inhibition (PPI). Although PPI does not require learning, its expression is regulated by higher cognitive processes. PPI deficits have been detected in several psychiatric conditions including schizophrenia where they coexist with cognitive deficits. A potential link between PPI expression and cognitive performance has therefore been suggested such that poor PPI may predict, or may be mechanistically linked to, overt cognitive impairments. A positive relationship between PPI and strategy formation, planning efficiency, and execution speed has been observed in healthy humans. However, parallel studies in healthy animals are rare. It thus remains unclear what cognitive domains may be associated with, or orthogonal to, sensory gating in the form of PPI in healthy animals. The present study evaluated a potential link between the magnitude of PPI and spatial memory
\end{abstract}

Daria Peleg-Raibstein and Singer Philipp are co-first author.

Benjamin K. Yee

benjamin.yee@trinity.oxon.org

1 Laboratory of Behavioural Neurobiology, Swiss Federal Institute of Technology Zurich, Schorenstrasse 16,

8603 Schwerzenbach, Switzerland

2 Laboratory of Translational Nutrition Biology, Swiss Federal Institute of Technology Zurich, Schorenstrasse 16, 8603 Schwerzenbach, Switzerland

3 Laboratory of Behavioral Neuroscience, Robert Stone Dow Neurobiology Laboratories, Legacy Research Institute, 1225 NE Second Avenue, Portland, OR 97232, USA performance by comparing two subgroups of animals differing substantially in baseline PPI expression (low-PPI vs highPPI) within a homogenous cohort of 100 male adult C57BL/6 mice. Assessment of spatial reference memory in the Morris water maze and spatial recognition memory in the Y-maze failed to reveal any difference between low-PPI and highPPI subjects. These negative findings contrast with our previous reports that individual difference in PPI correlated with sustained attention and working memory performance in C57BL/6 mice.

Keywords Recognition memory $\cdot$ Reference memory · Individual difference $\cdot$ Learning $\cdot$ Schizophrenia $\cdot$ Sensory gating $\cdot$ Startle reflex

Prepulse inhibition (PPI) of the acoustic startle reflex is an operational measure of sensorimotor gating. It refers to the reduction of the magnitude of the startle response when a weak prepulse precedes the startle-eliciting pulse stimulus (Graham, 1975; Hoffman \& Searle, 1965). Perception of the prepulse stimulus is believed to trigger an automatic preattentive gating mechanism that inhibits or 'gates' the processing of the subsequent pulse stimulus in order to protect the processing of the initial prepulse stimulus from distraction and interference by the pulse stimulus (Graham, 1992). While the expression of PPI is typically measured within sub-second time windows and does not require learning, it is nonetheless modulated by activities in brain regions that are also implicated in higher cognitive processes (Swerdlow, Geyer, \& Braff, 2001).

PPI is simple to measure compared with traditional neuropsychological tests (Geyer, \& Swerdlow, 2001). Importantly, it enjoys good test-retest reliability in both humans and animals (e.g., Abel, Waikar, Pedro, Hemsley, \& Geyer, 1998; 
Feifel, 1999; Ludewig, Geyer, Etzenberger, \& Vollenweider, 2002; Schwarzkopf, McCoy, Smith, Boutros, 1993). Hence, there is considerable interest in exploring the potential of PPI as a marker or a predictor of other overt cognitive and behavioral traits amongst both diseased and healthy individuals (Geyer, 2006a, b). A number of studies have investigated a potential link between PPI magnitude and cognitive performance in the general population. In healthy humans, there are reports that PPI positively correlated with strategy formation, planning efficiency, execution speed, and working memory performance (Bitsios \& Giakoumaki, 2005; Bitsios, Giakoumaki, Theou, \& Frangou, 2006; Csomor et al., 2008a; Giakoumaki, Bitsios, \& Frangou, 2006; Holstein et al., 2011). A positive relationship between PPI and cognitive performance is also suggested by the evaluation of clinical populations including patients with schizophrenia in which PPI deficiency often co-exists with other cognitive impairments (Hagan \& Jones, 2005; Young, Powell, Risbrough, Marston, \& Geyer, 2009). Given that cognitive deficiency in schizophrenia is now an accepted drug target independent of the psychotic symptoms (Laughren \& Levin, 2006, 2011), the relationship between PPI and cognition is of interest with respect to drug discovery for the treatment of cognitive symptoms in schizophrenia and related disorders (Geyer, 2006a, b).

PPI is highly translational since it is measured in very similar fashions in humans and rodents. The PPI paradigm therefore has been widely used to study the neurochemical and genetic basis of sensorimotor gating in animal models (Geyer, McIlwain, \& Paylor, 2002; Swerdlow et al., 2001). However, only a few studies have investigated the relationship between PPI expression and cognitive performance in normal animals. In our first attempt, we have recently examined in a homogeneous cohort of adult wild-type C57BL/6 mice the presence of a potential link between PPI magnitude and performance in standard tests of learning and memory including spatial reference memory, spatial working memory, and contextual fear conditioning (Singer et al., 2013). Apart from a highly specific positive correlation linking working memory performance with PPI induced by weak (but not strong) prepulse stimuli, no significant association between PPI and cognitive performance was discovered.

Here, to further explore the relationship between PPI and cognition and to test the suggestion that intrinsically low-PPI may be associated with poorer cognitive functions, we segregated a homogeneous cohort of 100 male adult C57BL/6 mice into two subgroups of subjects with low or high basal PPI levels. Cognitive performance of the two subgroups was then compared on spatial reference memory in the Morris water maze (including a challenge with new learning under high proactive interference) and spatial recognition memory in the Y-maze. In addition, spontaneous motor activity in the open field and anxiety-like behavior in the elevated plus maze were measured to control for confounding non-cognitive effects. The present segregation approach complements our previous study that solely relied on correlative analysis of individual differences to identify potential links between PPI expression and hippocampus-dependent spatial learning and memory function (Singer et al., 2013).

Our decision to adopt the current approach is to overcome an inherent limitation of the correlative approach in the study by Singer et al. (2013) and an earlier one by Bitanihirwe, Dubroqua, Singer, Feldon, \& Yee (2011). Namely, the limited sample size in each case arguably may limit the statistical power to detect correlative links especially when statistical corrections (for Type-I errors) may be deemed necessary to correct the family-wise Type-I error rate with the inclusions of multiple variables. In other words, a substantial increase in the number of subjects would be needed to provide robust statistical power, which could have severely limited our ability to perform multiple labor-intensive and time-consuming behavioral tests. By contrast, our current approach has enabled us to test our hypothesis based on a simple a priori comparison between two groups with a demonstrably robust difference in baseline PPI (an effect size $\eta_{\mathrm{p}}{ }^{2}=0.70$ ) as our independent variable. It has simplified interpretation as well as afforded us superior statistical power in answering whether a substantial baseline PPI difference entailed divergence in cognitive performance in specific tests. It should complement the correlative approach in the search of association between traits.

\section{Methods}

\section{Subjects}

A cohort of 100 12-week-old male C57BL/6NCrl mice were used. They were bred in the Laboratory of Behavioural Neurobiology (Swiss Federal Institute of Technology Zurich) from C57BL/6NCrl (strain code 027) breeding pairs originating from Charles River (Germany). They were born within one week of each other, weaned and sexed on postnatal day 21. All mice were housed with ad libitum access to food and water, in a temperatureand humidity-controlled $\left(22 \pm 1^{\circ} \mathrm{C}, 55 \pm 5 \%\right)$ vivarium maintained under a reversed 12/12-h light-dark cycle (lights on 1900-0700 h). All behavioral evaluations were conducted during the dark phase of the cycle. All procedures were conducted in accordance with the National Institutes of Health Guide for Care and Use of Laboratory Animals (1996) and had been approved by the Cantonal Veterinarian's Office of Zurich.

All animals were naive at the beginning of the PPI screening. Subsequently, 23 "high-PPI" and 23 "low-PPI" subjects were identified (see Results section ) and evaluated a week later in four behavioral tests in the order described below. Successive tests were separated by 2-3 days of recovery. 


\section{Prepulse inhibition (PPI) of the acoustic startle reflex}

The apparatus and procedures have been fully described elsewhere (Yee, Chang, \& Feldon, 2004a). In brief, four startle chambers for mice (SR-LAB, San Diego Instruments, San Diego, CA, USA) were used. During a PPI testing session, the animals were presented with a series of acoustic (white noise) stimuli comprising a mixture of four different types of trials: Pulse-alone trials, prepulse-alone trials, prepulse-pluspulse trials, and no-stimulus trials in which no discrete stimulus other than the constant background noise $\left(65 \mathrm{~dB}_{\mathrm{A}}\right)$ was presented. The intensity of the pulse stimulus was $120 \mathrm{~dB}_{\mathrm{A}}$ while prepulses of the following intensities were employed: $69,73,77,81$, and $85 \mathrm{~dB}_{\mathrm{A}}$, corresponding to $4,8,12,16$, and 20 decibel units above background, respectively. The duration of the pulse was $40 \mathrm{~ms}$, while a prepulse lasted $20 \mathrm{~ms}$. The stimulus onset asynchrony between the prepulse and pulse stimuli in prepulse-plus-pulse trials was $100 \mathrm{~ms}$.

The PPI test session consisted of three blocks of trials. In the first block, the animals were exposed to six consecutive pulse-alone trials in order to habituate and stabilize their startle response. PPI was measured in the middle block in which 12 series of discrete test trials were presented. Each series consisted of one trial of each of the following trial types: startle-alone, prepulse-plus-pulse of each of the five levels of prepulse, prepulse-alone of each of the five levels of prepulse, and no stimulus (background alone). The 12 discrete trials within each series were presented in a pseudorandom order, with a variable inter-trial interval averaging $15 \mathrm{~s}$ (ranging from 10 to $20 \mathrm{~s}$ ). The session was concluded with a final block of six consecutive pulse-alone trials similar to that presented in the first block. Whole body acceleration was measured by a stabilimeter on each and every trial within a $65-\mathrm{ms}$ response window (from the onset of the pulse in pulse-alone and prepulse-plus-pulse trials, and the onset of the prepulse on prepulse-alone trials, respectively). This output was referred to as reactivity score (in arbitrary units) and constituted the raw data from which a number of dependent measures were derived, as detailed below:

Startle reaction The mean startle reactivity across the 12 pulse-alone trials presented in the middle block was separately analyzed in order to evaluate the animals' startle reactivity.

Startle habituation To examine whether the startle reactivity habituated within the period of testing, the mean startle reactivity in the first and last block of pulse alone-trials was subjected to a separate analysis.

Prepulse-elicited reactivity A comparison between prepulse-alone trials at each level of intensity and nostimulus trials allowed the assessment of the direct reaction to the prepulse stimulus. Prepulse-elicited reactivity on each prepulse level was normalized against baseline reactivity on the no-stimulus trial by a difference score as performed by Yee et al. (2004a). In addition, the reaction to the prepulse was approximated by the linear component derived from the prepulse reactivity curve including no-stimulus trials (referred to as "prepulse steepness index").

Baseline reactivity Reactivity on no-stimulus trials was used as a measure of baseline reactivity in the absence of any discrete stimulus other than the background noise.

PPI The reactivity scores obtained on the 12 pulse-alone and prepulse-plus-pulse trials presented in the middle block were utilized to measure PPI. PPI was expressed as percent reduction of the startle reaction $(\% \mathrm{PPI})$ by the formula $\% \mathrm{PPI}=$ [(pulse-alone - prepulse-plus-pulse) $/$ pulse-alone $\times 100 \%$ ] as well as by the absolute reduction of the startle reaction as a function of increasing prepulse intensity (see Yee et al., 2004a). In the latter approach of PPI indexation, the PPI magnitude was indexed by the linear rate of which the startle reaction induced by the pulse stimulus is reduced by increasingly more intense prepulse stimuli. This method of indexing the magnitude of PPI is individually approximated by the linear component (namely, the fitted slope) of the startle reactivity curve as a function of increasing prepulse intensity (Yee, Chang, Pietropaolo, \& Feldon, 2005). This index is referred to as the "PPI steepness index" here.

\section{Anxiety-related behavior in the elevated plus maze}

Spontaneous anxiety-related behavior and unconditioned fear were evaluated by the elevated plus maze test. The apparatus and test procedures have been described in full detail elsewhere (Yee et al., 2004b). The test lasted 5 min during which the animal was allowed to explore freely undisturbed in the maze. Two anxiety-related measures were calculated: (i) percentage of time spent in the open arms [time in open arms / time in open and enclosed arms $\times 100 \%$ ], and (ii) percentage of entries made into the open arms [entries into open arms / entries into open and enclosed arms $\times 100 \%]$. In addition, spontaneous locomotor activity was measured by the cumulative distance travelled (in $\mathrm{m}$ ) in the entire maze. All raw data were generated by the Ethovision tracking software (Version 3.1, Noldus Technology, Wageningen, The Netherlands).

\section{Locomotor activity in the open field}

The apparatus consisted of four identical open-field arenas constructed from plastic laminated wood. Each arena measured $40 \times$ $40 \mathrm{~cm}$ in surface area and was surrounded on all sides by $35-\mathrm{cm}$ high walls. The experiment was conducted in a quiet testing room under diffused dim lighting (20 lux). The animals were tested in squads of four (one per arena). The allocation to the 
four arenas was counterbalanced between the two groups. A trial began by gently placing the animals in the center of the appropriate open field. They were then allowed to explore undisturbed in the open field for $1 \mathrm{~h}$. Afterwards, they were returned to the home cage and the arenas cleansed with diluted alcohol (10\%) and dried prior to the next squad. Locomotor activity was indexed by the distance travelled (in $\mathrm{m}$ ) expressed in successive 5-min bins. These data were calculated by the Ethovision (Version 3.1, Noldus Technology) tracking software.

\section{Spatial recognition memory in the Y-maze}

The Y-maze used to assess spatial recognition memory was identical to the one described by Pietropaolo et al. (2009). It could be placed in two distinct testing rooms (Rooms 1 and 2) each enriched with a unique set of visual extra-maze cues. Prior to the experiment, the animals were assigned two arms (start arm and familiar arm) to which they were exposed during the first phase of the test (sample phase). The remaining third arm constituted the novel arm and it was only accessible in the second phase (test phase). Allocation of arms (start, familiar, and novel) was counterbalanced within each experimental group. The maze floor was covered by a thin layer of sawdust to encourage exploration. The sawdust was never re-used between animals; and the sawdust was also replaced with fresh one between the two phases of the test to avoid any renmant of olfactory cues.

The sample phase began by releasing the animal from the distal end of the start arm facing the center of the maze. The animal was then given $5 \mathrm{~min}$ to freely explore the start and familiar arms. Timing started when the animal first entered the sample arm as detected by the Ethovision tracking software. In the subsequent test phase, the animal was again placed in the start arm and was allowed to explore all three arms for 3 min. Timing began upon detection of the animal in the central area of the maze by the Ethovision tracking software.

Two delay intervals ( 2 min and $3 \mathrm{~h}$ ) were examined on two consecutive days. On day 1 , the sample and test phases were separated by a delay of $2 \mathrm{~min}$, conducted in Room 1 . On day 2, a 3-h delay was imposed and the test was performed in Room 2. During the delay interval, the animals were either kept in waiting cages in the testing room (2-min delay) or were returned to the home cage (3-h delay).

Spatial novelty preference in the test phase was indexed by a discrimination ratio [time in novel arm / (time in novel + familiar arm)] as suggested by Bannerman et al. (2008). All data were generated by the Ethovision (Version 3.1, Noldus Technology) tracking software.

\section{Spatial reference memory and new learning in the water maze}

A detailed description of the apparatus and procedure has been provided elsewhere (Singer, Boison, Möhler, Feldon, \& Yee, 2009a). On the first day, the animals were pre-trained using a visually "cued" platform in order to familiarize them to swimming in the pool and climbing onto the platform. Each animal underwent three trials. The animals were allowed a maximum of $60 \mathrm{~s}$ to locate the platform on a given trial. If an animal failed to do so, it was guided to the platform by the experimenter and an escape latency of $60 \mathrm{~s}$ was scored on that trial. Across the three "cued" trials, the platform remained in a constant position, $25 \mathrm{~cm}$ from the center of the maze in the middle of one of four quadrants (NE, SE, SW, and NW). Assignment of the cued platform location was counterbalanced within in each group. A unique non-repeating sequence of release points (varied among N, E, S, and W) was randomly generated for each animal. The inter-trial interval (ITI) was $40 \mathrm{~s}$, of which the animal spent the first $20 \mathrm{~s}$ on the platform and the remaining $20 \mathrm{~s}$ in a waiting cage positioned next to the water maze.

Reference memory acquisition training commenced the next day. The platform was moved $90^{\circ}$ clockwise relative to its position in the cued trials. A new non-repeating sequence of starting points (N, E, S, and W) was generated daily for each animal. Four trials were performed daily, but they were separated into two sessions $3 \mathrm{~h}$ apart. The animals were returned to the home cage between the two sessions. Each session thus comprised two trials, separated by an ITI of $40 \mathrm{~s}$. Acquisition training continued for 3 days for a total of 12 trials. On day 4, the animals were subjected to a single probe trial in the first session when the escape platform was removed and the animals allowed to swim freely in the maze for $45 \mathrm{~s}$ after being randomly released from one of the four starting points. Next, new learning commenced in the second session on day 4 when the platform was moved to a new position - in the middle of the quadrant opposite to where it was positioned during acquisition training. Finally, new learning continued for another two trials on day 5 .

Performance during initial acquisition and new learning was measured by the latency (in sec) and path-length (in m) to reach the platform. Search accuracy during the probe test was indexed by percentage of time spent in the target quadrant, and percentage of path-length made inside the target quadrant.

\section{Statistical analysis}

All data were analyzed by parametric analysis of variance (ANOVA) with the between-subject factor basal PPI (high vs. low). Additional within-subject factors were included according to the nature of the considered dependent variables. To assist interpretation of the statistical outcomes, post-hoc analyses were performed based on the associated error terms taken from the overall ANOVA whenever appropriate. To better conform to the normality and variance homogeneity assumptions of parametric ANOVA, a natural logarithmic transformation (hereafter referred to as "ln-transformed") (see 
Csomor et al., 2008b) was applied to the reactivity data obtained in the PPI experiment except for \%PPI which was calculated based on the non-transformed data. All statistical analyses were carried out using SPSS for Windows (release 19, SPSS, Chicago, IL, USA) implemented on a PC running the Windows 7 operating system.

\section{Results}

\section{Segregation of subjects with high and low-PPI}

Based on the initial screening of the cohort of C57BL/6 male mice $(n=100)$, two subsets differing in baseline magnitude of PPI were identified. First, the cohort was trimmed with respect to four variables: (i) average baseline reactivity score (lntransformed) obtained across "no-stimulus" trials, (ii) average startle reactivity ( $n$-transformed) obtained in "pulse-alone" trials, (iii) average percentage PPI across all prepulse-pluspulse trials (as defined in the Procedures), and (iv) the alternative index of PPI - the "PPI steepness index" - which measured the steepness of the downward-sloping reactivity curve from pulse-alone trials to prepulse-plus-pulse trials with increasing prepulse intensity. Because our initial sample size was sufficiently large, we decided a priori that it is prudent to exclude subjects with the three highest and lowest scores in each of the four variables. It could minimize potential confounding effects of outlying subjects that lay at both extreme ends of the distribution. The trimming was to approximate the convention that the extreme $5 \%$ of the general population (in effect $6 \%$ here, with the top and bottom three extremes dropped) might be considered as outside the "norm" of the general population. Indeed, 15 out of the 22 mice excluded had at least one variable scoring beyond or very close to the $\pm 1.96 \times$ SD cut offs from the respective grand mean as expected from a normal Gaussian distribution.

Next, the remaining 78 subjects were then split according to the median of the two complementary variables (iii) and (iv) that index PPI. We did not rely on the common measure of $\%$ PPI alone because of fear that it can be distorted by baseline startle reactivity (see Csomor et al., 2008b). To this end, only animals receiving the same categorization for both variables were included in the final segregation, which yielded 23 "high-PPI" and 23 "low-PPI" subjects. It implied that 32 mice received inconsistent allocations between the two variables. The disagreement reflected partly the opposing influence of baseline startle on the two variables, as lower baseline startle tended to increase \%PPI but decrease the PPI steepness index. Thus, exclusion of subjects with inconsistent allocation ensured that our segregation was robust. The inclusion of the two complementary PPI indexes further ensured that the two groups were highly comparable in terms of startle baseline recorded on pulse-alone trials (Fig. 1) without any explicit attempt to match the baseline startle between the two subgroups. A few subjects received mixed allocation because they scored close to the median of the variables, and their exclusion should increase the effect size of the group difference in baseline PPI. The descriptive and inferential statistics comparing the "highPPI" and "low-PPI" subjects are as follows.

The expression of PPI A $2 \times 6$ (Basal PPI grouping $\times$ Prepulse intensity) ANOVA of the $l n$-transformed reactivity scores obtained in pulse-alone and prepulse-plus-pulse trials yielded a significant main effect of prepulse intensity $[F(5$, $\left.220)=310.66, p<.001, \eta_{p}{ }^{2}=.88\right]$. The prepulse stimulus was very effective in inhibiting the startle reaction elicited by the succeeding pulse stimulus, and the inhibition is proportional to the intensity of the prepulse (Fig. 1A). However, the rate of startle reduction as a function of prepulse intensity was clearly more pronounced in the high-PPI group. The difference led to the emergence of a significant group $\times$ prepulse intensity interaction $[F(5,220)=17.57, p<.001]$. Polynomial contrast analysis further revealed that the group $\times$ prepulse intensity interaction was the strongest in its linear component $[F(1,44)=41.84, p<.001$, $\eta_{p}{ }^{2}=.49$ ] (Fig. 1B). Post-hoc pairwise comparisons indicated that the startle reaction to the pulse stimulus was significantly lower in the high-PPI group when compared with the low-PPI group from prepulse intensity $>8 \mathrm{~dB}_{\mathrm{A}}$ above background. Furthermore, this group difference grew with increasing prepulse intensity with the inhibition of the startle reaction (i.e., PPI) approaching asymptote earlier, and at a lower level of inhibition, in the low-PPI group compared with the high-PPI group.

Parallel analysis of \%PPI by a $2 \times 5$ (Basal PPI grouping $\times$ Prepulse intensity) ANOVA essentially yielded a similar pattern of results (Fig. 1C). The general dependency of the PPI magnitude on the prepulse intensity (indexed by \%PPI) was again demonstrated by the significant effect of prepulse intensity $\left[F(4,176)=87.91, p<.001, \eta_{p}{ }^{2}=.67\right]$. Furthermore, $\%$ PPI was consistently weaker in the low PPI group regardless of prepulse intensity yielding a significant overall group difference $\left[F(1,44)=101.34, p<.001, \eta_{p}{ }^{2}=.70\right]$ while the group by prepulse intensity interaction was far from statistical significance $(p>.34)$. As expected, the percent transformation conferred additional power to the detection of group difference in PPI expression by comparison with the critical effect size (the linear component of the group $\times$ prepulse intensity interaction was $\eta_{p}{ }^{2}=.49$ ) obtained in the previous analysis.

Reactivity obtained on no-stimulus and prepulse-alone trials Apart from the expected divergence on PPI, the two groups also differed in terms of baseline reactivity obtained on no-stimulus trials and the direct reaction elicited by the prepulse stimulus (Fig. 1D). Baseline spontaneous reactivity was significantly higher in low-PPI than the high-PPI group $\left[F(1,44)=24.58, p<.001, \eta_{p}{ }^{2}=.36\right]$ as indicated by an oneway ANOVA of the mean reactivity ( $n$-transformed) recorded 
A

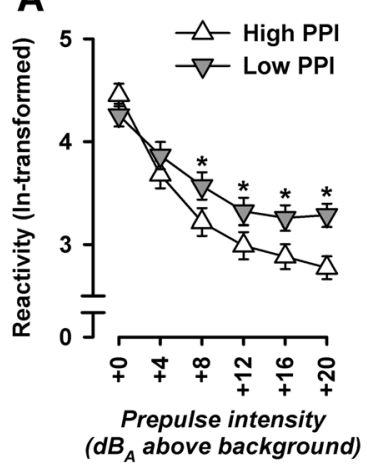

B

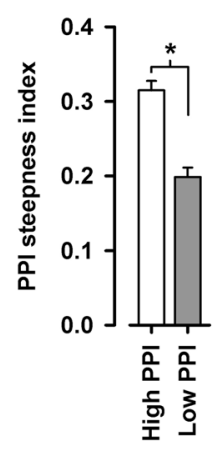

C

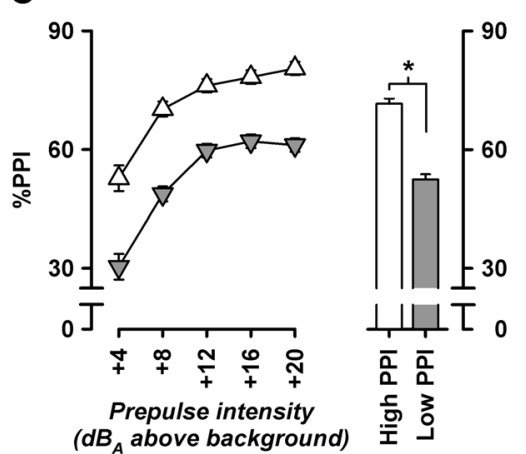

D

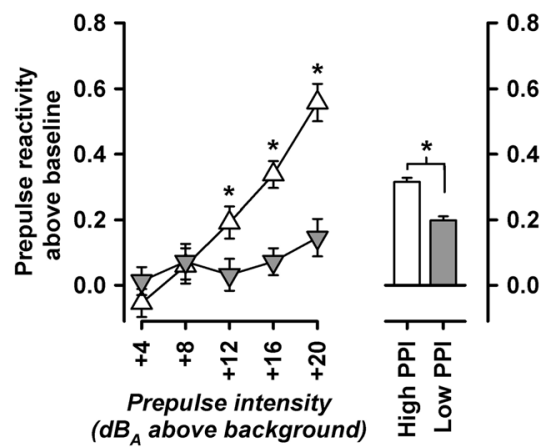

Fig. 1 Comparison of the high-PPI $(n=23)$ and low-PPI $(n=23)$ groups. (A) Reactivity scores (ln-transformed) obtained on pulse-alone and prepulse-plus-pulse trials. (B) Linear component derived from the data set shown in A. (C) \%PPI expressed as function of increasing prepulse intensity and as overall mean in the bar-plot on the right. (D) Prepulseelicited reactivity was indexed by a difference score (as deviation from no stimulus trials) which is also shown as a function of increasing prepulse intensity and as overall mean. Prepulse intensity is expressed as decibel units above the constant background (BG) of $65 \mathrm{~dB}_{\mathrm{A}}$. " +0 " refers to pulse-alone trials in which no prepulse was present. All values refer to mean \pm SE. * denotes a significant group difference $(p<.05)$ based on the respective ANOVA or according to subsequent post hoc comparisons on no-stimulus trials (high-PPI $=2.0$, low-PPI $=2.5$, standard error $(\mathrm{SE})=.1)$. To control for this difference in spontaneous reactivity, prepulse-elicited reactivity was indexed by a difference score that reflects the deviation from no-stimulus trials (Fig. 1D). This measure showed a clear increase with stronger prepulse intensity, and this increase appeared progressively weaker in the low-PPI relative to the high-PPI group. Consistent with these observations, a $2 \times 5$ (Basal PPI grouping $\times$ Prepulse intensity) ANOVA of the difference score revealed a significant main effect of group $\left[F(1,44)=9.04, p<.005, \eta_{p}{ }^{2}=\right.$ $.17]$, prepulse intensity $\left[F(4,176)=28.26, p<.001, \eta p^{2}=.39\right]$, and their interaction $\left[F(4,176)=13.56, p<.001, \eta_{p}{ }^{2}=.24\right]$. Additional polynomial contrast analysis showed that the interaction reached its highest significance level in the linear component $\left[F(1,44)=51.98, p<.001, \eta p^{2}=.54\right]$.

Lack of difference in startle habituation To examine whether the startle reaction habituated within the period of testing the first and last block of pulse-alone trials were subjected to a $2 \times 2$ (Basal PPI grouping $\times$ Blocks) ANOVA. It showed that the startle reaction did not significantly differ across blocks $(p>.3)$, implying that the animals' startle reaction was stable during the period of PPI assessment. The mean startle reaction (ln-transformed) on the two blocks was as follows: first block: high-PPI $=4.23$, low-PPI $=4.29, \mathrm{SE}=.14$; last block: high-PPI $=4.20$, low-PPI $=4.47, \mathrm{SE}=.15$.

The lack of clear startle habituation here was expected as we have repeatedly shown that C57BL/6 mice did not show reliable stable habituation (e.g., Pietropaolo, Singer, Feldon, \& Yee, 2008; Singer, Feldon, \& Yee, 2009b; Singer \& Yee, 2012; Yee et al., 2004a, 2005) in our laboratory. Singer et al. (2013) reported that while some subjects (C57BL/6 mice) showed habituation, a similar number showed sensitization. However, it should be noted that habituation is typically observed in other mouse strains and rodent species as well as humans.

\section{Comparable expression of baseline anxiety or locomotor activity}

The probability to enter and spend time in the open arms in the elevated plus maze provided an index of anxiety, and this expression was highly comparable between the two groups. Analysis of percentage open arm time (high-PPI $=26.0 \%$, low-PPI $=18.6 \%, \mathrm{SE}=3.9)$ and percentage open arm entries (high-PPI=31.4\%, low-PPI=29.7 \%, SE = 3.3) did not reveal any significant difference. Locomotor activity as measured by the cumulative distance travelled (in $\mathrm{m}$ ) was also comparable between the two groups (high-PPI $=11.20 \mathrm{~m}$, low-PPI $=$ $11.13 \mathrm{~m}, \mathrm{SE}=.5)$. The impression of a lack of difference in anxiety agreed with the open field test that had examined activity and spatial exploration over a much longer period of time. As shown in Fig. 2, the temporal profile of locomotor

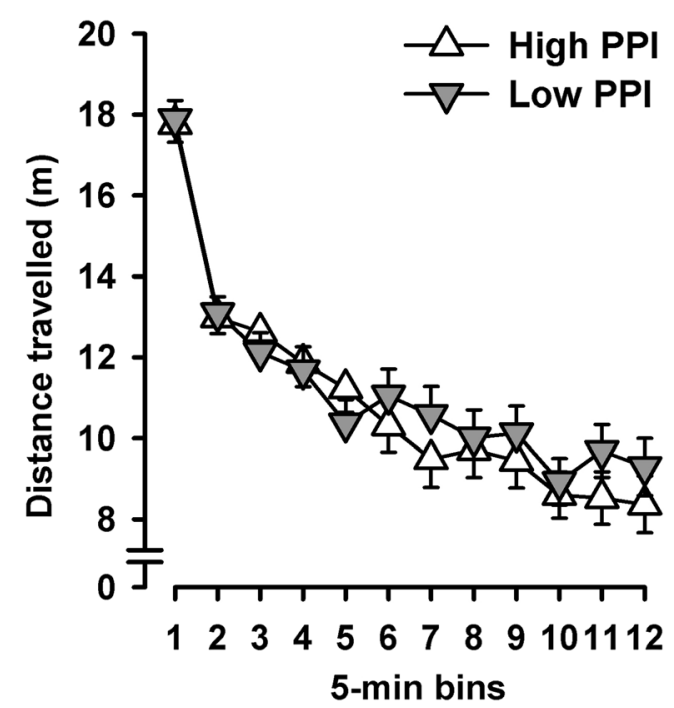

Fig. 2 Spontaneous locomotor activity in the open field expressed in 5min bins. All values refer to the mean $\pm \mathrm{SE}$ 
activity was nearly indistinguishable between the high-PPI and low-PPI groups, including the expression of locomotor habituation over time. A $2 \times 12$ (Basal PPI grouping $\times$ bins) ANOVA of the distance travelled per 5-min bin revealed only a significant main effect of bins $[F(11,484)=80.66, p<.001]$.

\section{Comparable expression of spatial recognition memory in the Y-maze}

Data of one high-PPI subject were lost since it had escaped from the Y-maze during test.

Test phase Irrespective of groups, the animals exhibited a delay-dependent preference for the novel arm (Fig. 3), which led to a significant main effect of delays $[F(1,43)=5.81$, $p<.05]$ in a $2 \times 2$ (Basal PPI grouping $\times$ Delays) ANOVA of the discrimination ratio. One-sample t-tests based on the pooled data of the two groups indicated that the preference was significantly above chance (i.e., discrimination ratio $>.5$ ) on the 2 -min delay condition $[t(44)=6.28, p<.001]$. The novelty preference was clearly weaker on the 3 -h delay condition while performance still maintained at above chance level $[t(44)=2.02, p=.0497]$.

Additional analysis of the absolute exploration times by a 2 $\times 2 \times 2$ (Basal PPI grouping $\times$ Delays $\times$ Arms) ANOVA revealed a similar pattern of results. Irrespective of group, the animals spent more time in the novel arm than in the familiar $\operatorname{arm}[F(1,43)=44.59, p<.001]$, and the magnitude of this bias was dependent on the delay as indicated by a significant Delays $\times$ Arms interaction $[F(1,43)=6.70, p<.05]$. Similar to the sample phase, the level of exploration (familiar and novel arms)

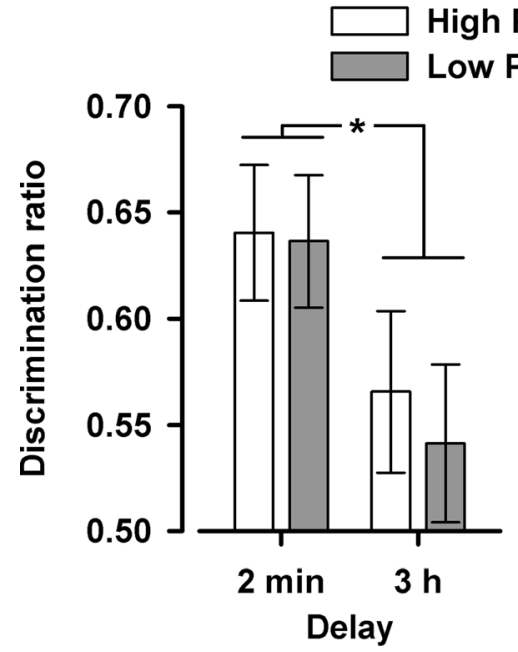

Fig. 3 Spatial familiarity judgment in the Y-maze. Novelty preference is indexed by a discrimination ratio based on time spent exploring the novel and familiar arms, namely, [novel / (novel + familiar)]. A ratio $>.5$ indicates a preference towards the novel over the familiar arm. $*$ denotes a significant delay effect $(p<.05)$ emerged in a $2 \times 2$ (Basal PPI grouping $\times$ Delays) ANOVA of the discrimination ratio. All values refer to the mean $\pm \mathrm{SE}$ generally decreased across delays $[F(1,43)=5.08, p<.05]$ but did not differ between groups $(F<1)$.

Sample phase The amount of time spent in the familiar arm generally decreased across the two delay conditions (Table 1), yielding a significant main effect of delays $[F(1,43)=7.68$, $p<.01]$ in a $2 \times 2$ (Basal PPI grouping $\times$ Delays) ANOVA of the time spent in the familiar arm. The exploration time appeared somewhat longer in the high-PPI group than the lowPPI group but this numerical difference, in the range of 6$10.7 \%$, was far from statistical significance.

\section{Comparable performance throughout the water maze test}

Cued test All animals rapidly acquired the escape response onto the visually cued platform across the three trials and performance on the cued test was highly comparable between groups (Fig. 4). Separate $2 \times 3$ (Basal PPI grouping $\times$ Trials) ANOVAs of escape latency and path-length yielded only a significant main effect of trials [latency: $F(2,88)=19.27, p<.001$; path-length: $F(2,88)=28.94, p<.001]$. The average swim speed was highly comparable between groups (high-PPI $=.16$, low-PPI $=.17, \mathrm{SE}=.004 \mathrm{~ms}^{-1}$ ).

Reference memory There was a gradual decrease in escape latency and path-length as training progressed, which was similarly observed in the two groups (Fig. 5A and B). A significant main effect of sessions [latency: $\mathrm{F}(5,220)=6.32$, $p<.001$, path-length $F(5,220)=8.61, p<.001]$ was revealed by separate $2 \times 6 \times 2$ (Basal PPI grouping $\times$ Sessions $\times$ Trials) ANOVAs. It was accompanied by a significant main effect trials [latency: $F(1,44)=10.25, p<.001$, path-length $F(1$, $44)=14.90, p<.001]$, further suggesting that performance improved across the two trials within a session. Neither the main effect of group nor any of its interactions attained statistical significance. Analysis of swim speed again failed to reveal any significant group difference. The average swim speed (in $\mathrm{ms}^{-1}$ ) was nearly identical to that obtained in the cued test: high-PPI $=.16$, low-PPI $=.17, \mathrm{SE}=.003$.

Table 1 Exploration times (in s) in the familiar and novel arms during the sample and test phases are shown separately for the two delay conditions $(2 \mathrm{~min}$ and $3 \mathrm{~h}$ ). All values refer to mean $\pm \mathrm{SE}$

Arm exploration (in s) in the Y-maze experiment

\begin{tabular}{lllll}
\hline Delay & PPI & Sample phase & \multicolumn{2}{l}{ Test phase } \\
\cline { 4 - 5 } & & Familiar arm & Familiar arm & Novel arm \\
\hline 2 min & High & $149.6 \pm 7.1$ & $46.0 \pm 3.6$ & $82.8 \pm 4.5$ \\
& Low & $135.2 \pm 6.9$ & $42.8 \pm 3.5$ & $76.2 \pm 4.4$ \\
$3 \mathrm{~h}$ & High & $129.7 \pm 4.8$ & $46.1 \pm 4.0$ & $65.6 \pm 5.4$ \\
& Low & $122.4 \pm 4.7$ & $53.8 \pm 3.9$ & $66.0 \pm 5.3$ \\
\hline
\end{tabular}


A
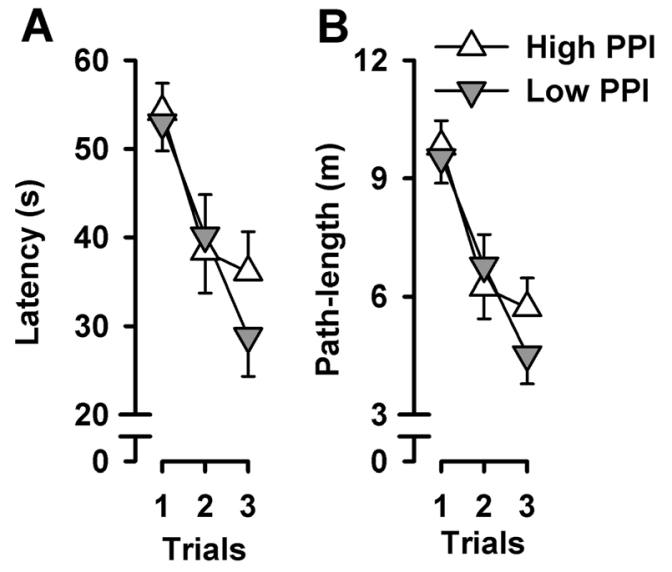

Fig. 4 Water maze cued test performance. Performance was measured by escape latency (A) and path-length (B). Both groups learned to locate and escape onto the visually cued escape platform across the three trials. All values refer to the mean $\pm \mathrm{SE}$

New learning As expected, the change in the platform location produced a general reduction in performance on the first session of new learning as evidenced by an increase in both latency (Fig. 5a) and path-length (Fig. 5B) to a level comparable to the initial training session. However, the animals quickly adapted their search strategy and reached within two sessions a level of performance comparable to that seen at the end of the acquisition phase. The rapid new learning was evidenced by the significant main effect of sessions in a $2 \times 2$ $\times 2$ (Basal PPI grouping $\times$ Sessions $\times$ Trials) ANOVA of latency $[F(1,44)=52.79, p<.001]$ or path-length: $[F(1,44)$ $=52.34, p<.001]$. As seen before, performance generally improved from the first to the second trial within a session [latency: $F(1,44)=7.10, p<.05$; path-length: $F(1,44)=$ $8.03, p<.01]$. This pattern of results was similarly observed in both groups. Likewise, the two groups swam at a comparable speed. The average swim speed (in $\mathrm{ms}^{-1}$ ) of each group was: high-PPI $=.16$, low-PPI $=.17, \mathrm{SE}=.006$.

Probe test Search accuracy as indexed by the percentage of time (Fig. 5C) and percentage of path-length (Fig. 5D) spent inside the target quadrant did not differ between groups. There was an overall search bias for the target quadrant yielding a significant main effect of quadrants in two separate $2 \times 4$ (Basal PPI grouping $\times$ Quadrants) ANOVAs of percentage time $[F(3,132)=31.85, p<.001]$ and percentage path-
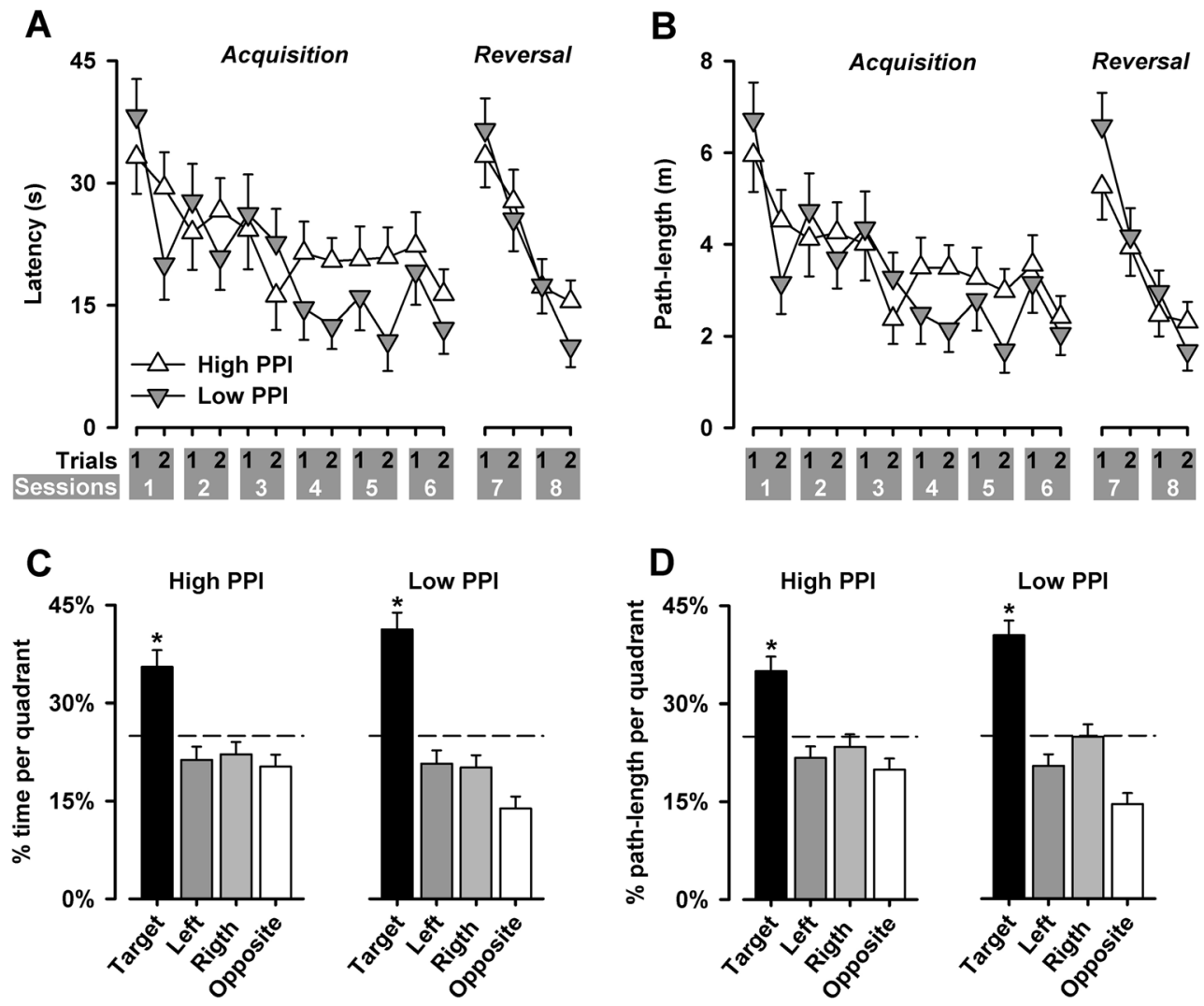

Fig. 5 Spatial reference memory and new learning. The two groups showed similar escape performance on the initial acquisition phase and learned the novel platform location in the new phase at a comparable rate in terms of latency (A) as well as path-length (B). Likewise, search accuracy during the probe test did not differ between the two groups.

Both groups preferentially searched in the target quadrant as expressed by percentage time $(\mathbf{C})$ and percentage path-length $(\mathbf{D})$ per quadrant. * denotes that the preference for the target quadrant was significantly above chance (i.e., $>25 \%$ as indicated by the dashed line) according to onesample t-tests $(p<.05)$. All values refer to mean \pm SE 
length $[\mathrm{F}(3,132)=32.33, p<.001]$ per quadrant. Supplementary one-sample t-tests confirmed that the preference for the target quadrant (in percentage time and percentage path-length) was significantly above chance level (i.e. $>25 \%$ ) in each group $(p$ 's $<.05)$. We also restricted the analysis to the first $15 \mathrm{~s}$ in order to examine whether the two groups potentially differed in beginning of the probe test and again did not find any statistical evidence for a significant group difference (data not shown).

\section{Discussion}

Despite the substantial difference in the magnitude of PPI expression between low and high PPI subjects that measured an effect size of $0.7\left(\eta_{p}{ }^{2}\right)$ in terms of \%PPI, the two groups remained highly comparable in all measures of spatial cognition in the present study. These negative findings in the memory tests were paralleled by the absence of any difference between low and high PPI animals in the open field and the elevated plus maze test of anxiety. In addition, as we have reported elsewhere, the low-PPI compared with the high-PPI subjects evaluated here were marked by significant neurochemical differences. Postmortem HPLC analysis showed that the levels of dopamine and its metabolites in the nucleus accumbens and medial prefrontal cortex of the low-PPI subjects were significantly lower than in the high-PPI subjects (Peleg-Raibstein et al, 2013). This neurochemical difference might underlie the stronger development of behavioral sensitization to amphetamine observed in the low-PPI subjects (Peleg-Raibstein et al, 2013).

In the Morris water maze, low and high PPI subjects acquired the location of the escape platform at a similar pace and showed comparable long-term retention of the newly acquired reference memory as indicated by the nearly identical search preference for the target quadrant in the probe test conducted $24 \mathrm{~h}$ after the last day of acquisition. The absence of an effect here is consistent with the negative result of a previous study which failed to detect any significant correlation between individual PPI levels and reference memory performance within a homogeneous cohort of 23 naïve C57BL/6 mice (Singer et al., 2013). Even when the animals were further challenged to acquire a second position of the escape platform under the negative influence of proactive interference from the previously acquired platform (i.e., new learning following the initial acquisition), performance was still highly comparable between low and high PPI subjects. These findings strengthen the impression that reference memory performance within the normal population of adult male C57BL/6 mice cannot be predicted by baseline PPI levels.

Likewise, the Y-maze test also did not reveal any difference between the high- and low-PPI subjects. Both groups showed a strong and comparable preference for the novel over the familiar arm when the demand on memory retention was low (2-min delay); and their performance remained very similar when the overall preference for the novel arm was weakened by extending the retention period to $3 \mathrm{~h}$.

The lack of difference between high- and low-PPI subjects on these two well-known hippocampus-dependent tests of spatial learning (e.g., Bannerman et al., 2004; Sanderson et al., 2007) is consistent with our previous study, which showed that individual difference in PPI expression also failed to predict the expression of contextual fear acquired through Pavlovian conditioning (Singer et al., 2013), a test that similarly depends on the hippocampus (e.g., Phillips \& LeDoux, 1992; Seldon, Everitt, Jarrard, \& Robbins, 1991). Hence, individual difference in spatial memory is largely orthogonal to variation in PPI expression among healthy male C57BL/6 mice.

Against the above conclusion, our recent demonstration of a positive correlation between PPI and spatial working memory performance in C57BL/6 mice (Singer et al., 2013) may seem to be an exceptional finding. However, it should be noted that the reported statistical link was only evident when the memory load was relatively low because the test only required the animal to remember one specific location on a given trial. Furthermore, the statistical link was restricted to PPI obtained with the weakest prepulses. It suggests that PPI is unlikely to reliably predict the retention capacity of the working memory buffer in mice. In keeping with this interpretation, no evidence for a potential link between basal PPI and memory capacity has been forthcoming in healthy humans, even though the assessment and conception of working memory do differ somewhat between humans and rodents (Baddeley, 2012; Dudchenko, 2004; Honig, 1978; Olton, Becker, \& Handelmann, 1979). Instead, poor PPI in human volunteers has been repeatedly shown to predict poor strategy formation and slow execution time in spatial working memory tests (Bitsios \& Giakoumaki, 2005; Bitsios et al., 2006; Csomor et al., 2008a ; Giakoumaki et al., 2006; Holstein et al., 2011).

In line with the emphasis on executive functioning based on the human data, we have previously identified a significant positive correlation between PPI and sustained attention or vigilance in a visual two-choice discrimination task in a different cohort of C57BL/6 mice (Bitanihirwe et al., 2011). A potential link between PPI and attention is further supported by several reports showing that attentional processes may critically modify the expression of PPI (Dawson, Hazlett, Filion, Nuechterlein, \& Schell, 1993; Scholes \& Martin-Iverson, 2009; Scholes \& Martin-Iverson, 2010). Hence, attention and executive functions could be important mediating factors that underlie the co-existence of PPI deficiency and memory deficiency in specific diseases such as schizophrenia (Geyer, 2006b).

Indeed, the extent to which basal PPI levels may predict cognitive performance in schizophrenia patients remains unclear and may not be a reliable tool to aid clinical diagnosis 
(Young, Powell, Risbrough, Marston, \& Geyer, 2009). One notable study failed to detect any correlation between PPI and cognitive performance in a large cohort of schizophrenia patients tested across a comprehensive battery of cognitive tests (Swerdlow et al., 2006). Furthermore, cognitive deficits are not always associated with low PPI. Thus, patients with Alzheimer's disease who show memory deficits do not necessarily exhibit poor PPI levels compared with healthy controls (Hejl, Glenthøj, Mackeprang, Hemmingsenm, \& Waldemar, 2004; Ueki, Goto, Sato, Iso, \& Morita, 2006). Likewise, PPI does not seem to decrease with normal aging while cognitive performance shows a clear age-dependent decline in the general population (Ellwanger, Geyer, \& Braff, 2003) leading to the suggestion that age-related cognitive dysfunction is orthogonal to PPI (Young et al., 2009).

Hence, it would seem premature to rely on individual differences in PPI to predict, qualitatively or quantitatively, memory performance in healthy C57BL/6 mice whilst its correlative links with other attentional and executive functions may merit more serious consideration. Moreover, it remains to be ascertained whether our findings that have been gathered in this specific inbred mouse strain could be generalized to other strains or species. Indeed, the individual differences observed here and in our previous studies using in-bred C57BL/ 6 mice likely reflect the influences of environmental and/or developmental factors rather than genetic variations. In order to better capture the situations in the normal human population, it is imperative to extend our investigation to outbred strains (as well as other inbred strains) in combination with gene mapping technology (e.g., Flint and Mott, 2008) to undercover potential gene-environment interaction.

Acknowledgments The present study was supported by the ETH Zurich. The authors are grateful to Peter Schmid, Hannes Sigrist, and the practicum students for technical assistance, and to the animal husbandry staff at the Laboratory of Behavioral Neurobiology for the breeding and maintenance of the animals used in the experiments. The authors have no conflict of interest to declare. DPR, SP, JF and BKY designed the experiments. DPR, SP and BKY supervised and performed the experiments described. SP and BKY finalized and inferred all statistical analyses and prepared the manuscript for publication. DPR and SP are co-first authors.

\section{References}

Abel, K., Waikar, M., Pedro, B., Hemsley, D., \& Geyer, M. (1998). Repeated testing of prepulse inhibition and habituation of the startle reflex: A study in healthy human controls. Journal of Psychopharmacology, 12, 330-337.

Baddeley, A. (2012). Working memory: Theories, models, and controversies. Annual Review of Psychology, 63, 1-29.

Bannerman, D.M., Niewoehner, B., Lyon, L., Romberg, C., Schmitt, W.B., Taylor, A., ... Rawlins, J.N.P. (2008). NMDA receptor subunit NR2A is required for rapidly acquired spatial working memory but not incremental spatial reference memory. The Journal of Neuroscience, 28, 3623-3630.
Bannerman. D.M., Rawlins, J.N., McHugh, S.B., Deacon, R.M., Yee, B.K., Bast, T., ... Feldon, J. (2004). Regional dissociations within the hippocampus-memory and anxiety. Neuroscience \& Biobehavioral Reviews, 28, 273-283.

Bitanihirwe, B. K., Dubroqua, S., Singer, P., Feldon, J., \& Yee, B. K. (2011). Sensorimotor gating and vigilance-dependent choice accuracy: A within-subject correlative analysis in wild-type C57BL/6 mice. Behavioural Brain Research, 217, 178-187.

Bitsios, P., \& Giakoumaki, S. G. (2005). Relationship of prepulse inhibition of the startle reflex to attentional and executive mechanisms in man. International Journal of Psychophysiology, 55, 229-241.

Bitsios, P., Giakoumaki, S. G., Theou, K., \& Frangou, S. (2006). Increased prepulse inhibition of the acoustic startle response is associated with better strategy formation and execution times in healthy males. Neuropsychologia, 44, 2494-2499.

Csomor, P. A., Stadler, R. R., Feldon, J., Yee, B. K., Geyer, M. A., \& Vollenweider, F. X. (2008a). Haloperidol differentially modulates prepulse inhibition and 50 suppression in healthy humans stratified for low and high gating levels. Neuropsychopharmacology, 33, 497-512.

Csomor, P. A., Yee, B. K., Vollenweider, F. X., Feldon, J., Nicolet, T., \& Quednow, B. B. (2008b). On the influence of baseline startle reactivity on the indexation of prepulse inhibition. Behavioral Neuroscience, 122, 885-900.

Dawson, M. E., Hazlett, E. A., Filion, D. L., Nuechterlein, K. H., \& Schell, A. M. (1993). Attention and schizophrenia: Impaired modulation of the startle reflex. Journal of Abnormal Psychology, 102, 633-641.

Dudchenko, P. A. (2004). An overview of the tasks used to test working memory in rodents. Neuroscience \& Biobehavioral Review, 28, 699-709.

Ellwanger, J., Geyer, M. A., \& Braff, D. L. (2003). The relationship of age to prepulse inhibition and habituation of the acoustic startle response. Biological Psychology, 62, 175-195.

Feifel, D. (1999). Individual differences in prepulse inhibition of startle as a measure of individual dopamine function. Behavioral Neuroscience, 113, 1020-1029.

Flint, J., \& Mott, R. (2008). Applying mouse complex-trait resources to behavioural genetics. Nature, 456, 724-727.

Geyer, M. A. (2006a). Are cross-species measures of sensorimotor gating useful for the discovery of procognitive co-treatments for schizophrenia? Dialogues in Clinical Neuroscience, 8, 9-16.

Geyer, M. A. (2006b). The family of sensorimotor gating disorders: Comorbidities or diagnostic overlaps? Neurotoxicity Research, 10, 211-220.

Geyer, M. A., McIlwain, K. L., \& Paylor, R. (2002). Mouse genetic models for prepulse inhibition: An early review. Molecular Psychiatry, 7, 1039-1053.

Geyer, M.A., \& Swerdlow, N.R. (2001). Measurement of startle response, prepulse inhibition, and habituation. Current Protocols in Neuroscience, Chapter 8, Unit 8.7

Giakoumaki, S. G., Bitsios, P., \& Frangou, S. (2006). The level of prepulse inhibition in healthy individuals may index cortical modulation of early information processing. Brain Research, 1078, 168 170 .

Graham, F. K. (1975). The more or less startling effects of weak prestimulation. Psychophysiology, 12, 238-248.

Graham, F. K. (1992). Attention and information processing in infants and adults: perspectives from human and animal research. In B. A. Campell, H. Hayne, \& R. Richardson (Eds.), Attention: The heartbeat, the blink, and the brain (pp. 3-29). Hillsdale, NJ: Lawrence Erlbaum Associates.

Hagan, J. J., \& Jones, D. N. (2005). Predicting drug efficacy for cognitive deficits in schizophrenia. Schizophrenia Bulletin, 31, 830-853. 
Hejl, A. M., Glenthøj, B., Mackeprang, T., Hemmingsenm, R., \& Waldemar, G. (2004). Prepulse inhibition in patients with Alzheimer's disease. Neurobiology of Aging, 25, 1045-1050.

Hoffman, H. S., \& Searle, J. L. (1965). Acoustic variables in the modification of startle reaction in the rat. Journal of Comparative and Physiological Psychology, 60, 53-58.

Holstein, D. H., Csomor, P. A., Geyer, M. A., Huber, T., Bruggerm, N., Studerusm, E., \& Vollenweider, F. X. (2011). The effects of sertindole on sensory gating, sensorimotor gating, and cognition in healthy volunteers. Journal of Psychopharmacology, 25, 1600 1613.

Honig, W. K. (1978). Studies of working memory in the pigeon. In S. H. Hulse, H. F. Fowler, \& W. K. Honig (Eds.), Cognitive processes in animal behavior (pp. 211-248). Hillsdale, NJ: Lawrence Erlbaum Associates.

Laughren, T., \& Levin, R. (2006). Food and Drug Administration perspective on negative symptoms in schizophrenia as a target for a drug treatment claim. Schizophrenia Bulletin, 232, 220-222.

Laughren, T., \& Levin, R. (2011). Food and Drug Administration commentary on methodological issues in negative symptom trials. Schizophrenia Bulletin, 37, 255-256.

Ludewig, K., Geyer, M. A., Etzensberger, M., \& Vollenweider, F. X. (2002). Stability of the acoustic startle reflex, prepulse inhibition, and habituation in schizophrenia. Schizophrenia Research, 55, 129137.

Olton, D. S., Becker, J. T., \& Handelmann, E. (1979). Hippocampus, space and memory. Behavioral and Brain Sciences, 2, 313-366.

Peleg-Raibstein, D., Hauser, J., Llano Lopez, L. H., Feldon, J., Gargiulo, P. A., \& Yee, B. K. (2013). Baseline prepulse inhibition expression predicts the propensity of developing sensitization to the motor stimulant effects of amphetamine in C57BL/6 mice. Psychopharmacology (Berl), 225, 341-352.

Phillips, R. G., \& LeDoux, J. E. (1992). Differential contribution of amygdala and hippocampus to cued and contextual fear conditioning. Behavioral Neuroscience, 106, 274-285.

Pietropaolo, S., Singer, P., Feldon, J., \& Yee, B. K. (2008). The postweaning social isolation in C57BL/6 mice: Preferential vulnerability in the male sex. Psychopharmacology (Berl), 197, 613-628.

Pietropaolo, S., Sun, Y., Li, R., Brana, C., Feldon, J., \& Yee, B. K. (2009). Limited impact of social isolation on Alzheimer-like symptoms in a triple transgenic mouse model. Behavioral Neuroscience, 123, 181195.

Sanderson, DJ., Gray, A., Simon, A., Taylor, A,M., Deacon, R,M,. Seeburg, P,H., ... Rawlins, J.N.P. (2007). Deletion of glutamate receptor-A (GluR-A) AMPA receptor subunits impairs one-trial spatial memory. Behavioral Neuroscience, 121, 559-569.

Scholes, K. E., \& Martin-Iverson, M. T. (2009). Relationships between prepulse inhibition and cognition are mediated by attentional processes. Behavioural Brain Research, 205, 456-467.

Scholes, K. E., \& Martin-Iverson, M. T. (2010). Disturbed prepulse inhibition in patients with schizophrenia is consequential to dysfunction of selective attention. Psychophysiology, 47, 223-235.
Schwarzkopf, S. B., McCoy, L., Smith, D. A., \& Boutros, N. N. (1993). Test-retest reliability of prepulse inhibition of the acoustic startle response. Biological Psychiatry, 34, 896-900.

Seldon, N., Everitt, B., Jarrard, L., \& Robbins, T. W. (1991). Complimentary roles for the amygdala and hippocampus in aversive conditioning to explicit and contextual cues. Neuroscience, 42, 335350.

Singer, P., Boison, D., Möhler, H., Feldon, J., \& Yee, B. K. (2009a). Deletion of glycine transporter 1 (GlyT1) in forebrain neurons facilitates reversal learning: Enhanced cognitive adaptability? Behavioral Neuroscience, 123, 1012-1027.

Singer, P., Feldon, J., \& Yee, B. K. (2009b). Are DBA/2 mice associated with schizophrenia-like endophenotypes? A behavioural contrast with C57BL/6 mice. Psychopharmacology (Berl), 206, 677-698.

Singer, P., Hauser, J., Llano Lopez, L., Peleg-Raibstein, D., Feldon, J., Gargiulo, P. A., \& Yee, B. K. (2013). Prepulse inhibition predicts working memory performance whilst startle habituation predicts spatial reference memory retention in C57BL/6 mice. Behavioural Brain Research, 242, 166-177.

Singer, P., \& Yee, B. K. (2012). Reversal of scopolamine-induced disruption of prepulse inhibition by clozapine in mice. Pharmacology Biochemistry \& Behavior, 101, 107-114.

Swerdlow, N. R., Geyer, M. A., \& Braff, D. L. (2001). Neural circuit regulation of prepulse inhibition of startle in the rat: Current knowledge and future challenges. Psychopharmacology (Berl), 156, 194 215.

Swerdlow, N. R., Light, G. A., Cadenhead, K. S., Sprock, J., Hsieh, M. H., \& Braff, D. L. (2006). Startle gating deficits in a large cohort of patients with schizophrenia: Relationship to medications, symptoms, neurocognition, and level of function. Archives of General Psychiatry, 63, 1325-1335.

Ueki, A., Goto, K., Sato, N., Iso, H., \& Morita, Y. (2006). Prepulse inhibition of acoustic startle response in mild cognitive impairment and mild dementia of Alzheimer type. Psychiatry and Clinical Neurosciences, 60, 55-62.

Yee, B. K., Chang, T., Pietropaolo, P., \& Feldon, J. (2005). The expression of prepulse inhibition of the acoustic startle reflex as a function of three pulse stimulus intensities, three prepulse stimulus intensities, and three levels of startle responsiveness in C57BL6/J mice. Behavioural Brain Research, 163, 265-276.

Yee, B. K., Chang, D. L., \& Feldon, J. (2004a). The Effects of dizocilpine and phencyclidine on prepulse inhibition of the acoustic startle reflex and on prepulse-elicited reactivity in C57BL6 mice. Neuropsychopharmacology, 29, 1865-1877.

Yee, B. K., Hauser, J., Dolgov, V. V., Keist, R., Mohler, H., Rudolph, U., \& Feldon, J. (2004b). GABA receptors containing the alpha5 subunit mediate the trace effect in aversive and appetitive conditioning and extinction of conditioned fear. European Journal of Neuroscience, 20, 1928-1936.

Young, J. W., Powell, S. B., Risbrough, V., Marston, H. M., \& Geyer, M. A. (2009). Using the MATRICS to guide development of a preclinical cognitive test battery for research in schizophrenia. Pharmacology \& Therapeutics, 122, 150-202. 